\title{
Evitación y psicopatología: Un estudio a través de una tarea experimental y su relación con medidas de autoinforme
}

\author{
Santiago Barajas \\ Hospital Universitario de Guadalajara (SESCAM), Guadalajara, España
}

\begin{abstract}
Resumen: En el estudio de la evitación en psicopatología destacan tres dimensiones: cognitiva, conductual y experiencial. Pero su investigación se ha realizado básicamente a través de medidas de autoinforme. En este trabajo se diseñó una tarea de evitación, basada en la presentación de estímulos visuales del IAPS (International Affective Picture System; Lang, Bradley y Cuthbert, 2005), y se estudió su relación con las siguientes medidas: CBAS, AAQ-II, MAAS, BAI y BDI-II. Se empleó una muestra clínica de pacientes con trastornos de ansiedad, trastornos depresivos y trastorno límite de la personalidad $(N=100)$, y una muestra de población general $(N=100)$. Los resultados indican que el grupo clínico muestra un nivel significativamente mayor de evitación que el grupo control en la tarea diseñada. En ambos grupos, existe una correlación entre dicha evitación y la sintomatología de ansiedad y depresión. Se discuten las implicaciones clínicas de estos hallazgos y se ofrecen recomendaciones para estudios futuros.
\end{abstract}

Palabras clave: Evitación; tarea experimental; IAPS; ansiedad; depresión; mindfulness.

Avoidance and psychopathology: A study through an experimental task and its relation with self-report measures

\begin{abstract}
When studying avoidance in psychopathology, three dimensions stand out: cognitive, behavioral and experiential. However, research has been conducted primarily using self-report measures. In this study, an avoidance task was designed that consisted of presenting visual stimuli from the IAPS (International Affective Picture System. Lang, Bradley and Cuthbert, 2005), and studying relationships with the following measures: CBAS, AAQ-II, MAAS, BAI y BDI-II. The study was conducted with a clinical sample of patients with anxiety disorders, depressive disorders and borderline personality disorder $(\mathrm{N}=100)$, and a general population sample $(\mathrm{N}=100)$. The results revealed significantly higher avoidance levels in the clinical sample than in the control group when performing the task. In both groups, a correlation was found between avoidance and the symptomatology of anxiety and depression. The clinical implications of these findings are discussed and recommendations for future investigation are given.
\end{abstract}

Keywords: Avoidance; experimental task; IAPS; anxiety; depression; mindfulness.

\section{Introducción}

La evitación juega un papel fundamental en la psicopatología. Seguramente, en la mayor parte de las personas con un trastorno mental podríamos identificar alguna forma relevante de evitación (cognitiva, conductual, experiencial), bien por su frecuencia o por su intensidad, que le genere problemas en su vida cotidiana. Así, po-

Recibido: 18 junio 2014; aceptado: 8 octubre 2014.

Correspondencia: Santiago Barajas, Hospital Universitario de Guadalajara (SESCAM), c/ Donante de Sangre s/n, 19002 Guadalajara, España. Correo-e: sbarajas@sescam.jccm.es dríamos pensar en un caso de trastorno depresivo mayor, en el que la persona evita el contacto con los demás, bien para no ver que los demás manifiestan un estado de ánimo "mucho mejor" que el propio, bien por evitar recibir comentarios negativos acerca del aspecto o actitud propios, etc. Podríamos también pensar en un caso de trastorno de angustia con agorafobia en el que se evitan determinadas situaciones, bien pudiera ser por evitar que los demás le observen y detecten sus síntomas de ansiedad, bien por evitar reexperimentar determinadas sensaciones fisiológicas desagradables, o bien por evitar enfrentarse con normalidad a dichas situaciones realizando 
conductas de seguridad tales como llevar ansiolíticos, ir acompañado, etc. En un caso de trastorno límite de la personalidad, la persona podría estar llevando a cabo conductas autolesivas como forma de evitar afrontar situaciones que le son desafiantes o como escape de pensamientos dolorosos, pero igualmente podría ponerse a imaginar sucesos futuros poco realistas dadas las circunstancias, evitando así afrontar los problemas reales que están aconteciendo en el presente. En un caso de abuso de alcohol o cualquier otra droga, podría plantearse un componente de evitación hacia tomar conciencia de determinados problemas personales en los que uno debería tomar un papel activo asumiendo su responsabilidad para poder cambiarlos. O por qué no, en un caso de esquizofrenia, las ideas delirantes de perjuicio podrían plantearse desde un punto de vista funcional como una forma de evitación extrema ante determinadas experiencias emocionales traumáticas vividas, o ante situaciones que se experimentan como altamente estresantes y frente a las cuales la persona no dispone de habilidades de afrontamiento adecuadas. La lista de posibles ejemplos podría ser tan larga como el número de personas en busca de tratamiento psicológico.

Aunque es evidente que en determinadas circunstancias una conducta de evitación resultaría adaptativa (p.ej., un riesgo claro para la salud y/o para la integridad física, cualquier situación que dispare una intensa sintomatología y ante la que la persona no disponga de otra estrategia de afrontamiento, etc.), lo cierto es que en la mayoría de los casos sólo suele ser efectiva en el corto plazo. Campos, Iraurgui, Páez y Velasco (2004), tras un estudio de meta-análisis afirman que, aunque el afrontamiento de evitación es frecuente en el mismo momento de aparición de un hecho estresante y sirve para minimizar la respuesta afectiva ya que ayuda a coordinar acciones y demandas del entorno, se puede concluir que se asocia de forma moderada a una peor regulación afectiva, depresión, ansiedad y en niños y adolescentes a más problemas de conducta. Respecto a la evitación cognitiva, Ottaviani et al. (2014) encuentran apoyo para el planteamiento de que la preocupación tiene la función adaptativa de mantener bajo control la activación fisiológica asociada con la ansiedad en controles sanos, pero sugieren que la preocupación no es una actitud funcional cuando se convierte en una respuesta automática y patológica.

Tradicionalmente se consideró la evitación dentro de la psicopatología de la ansiedad, donde el paradigma lo constituían las fobias y las conductas motoras de evitación. Posteriormente, la evitación ha adquirido un notable auge en el estudio de la depresión, especialmente de la mano de la terapia de Activación Conductual (Jacobson, Martell y Dimidjian, 2001; Kanter, Busch y Rusch,
2011; Martell, Addis y Jacobson, 2001; Martell, Dimidjian y Herman-Dunn, 2013). Más recientemente el papel de la evitación también está siendo objeto de estudio y se está estableciendo su relevancia en el campo de los trastornos psicóticos (Vilardaga, Hayes, Atkins, Bresee y Kambiz, 2013), donde se ha visto que evitar las alucinaciones auditivas supone un esfuerzo infructuoso y empeora el malestar causado por ellas (Morris, Johns y Oliver 2013; Pérez-Álvarez, García-Montes, Perona-Garcelán y Vallina-Fernández, 2008).

El afrontamiento evitativo es más empleado en depresivos que en sujetos normales, siendo un dato que se repite a través de diferentes países y culturas (Hernangómez y Vázquez, 2005). Por ejemplo, en un estudio prospectivo de diez años y con una muestra de 1211 personas de entre 55 y 65 años, Holahan, Moos, Holahan, Brennan y Schutte (2005) encontraron que el afrontamiento de evitación estaba asociado con experimentar más estresores vitales tanto agudos como crónicos cuatro años después. En dicho estudio también hallaron una relación entre el afrontamiento de evitación y la presencia de síntomas depresivos diez años después, incluso después de controlar el efecto de los síntomas depresivos en la línea base. Los efectos de la evitación conductual también han sido documentados, no sólo sobre la depresión, sino sobre la psicopatología en general (Kashdan, Barrios, Forsyth y Steger, 2006).

La evitación en su modalidad cognitiva también tiene gran relevancia y puede implicar una amplia variedad de formas tales como, intentar intencionadamente suprimir pensamientos o recuerdos, la rumiación de pensamientos, los esfuerzos para disociarse o separarse de las emociones asociadas a experiencias negativas, y el adoptar una perspectiva de tercera persona respecto a los recuerdos dolorosos. En personas depresivas por ejemplo, se ha comprobado que emplean como estrategia habitual la supresión de pensamientos, aunque generalmente suele ser evaluada por ellos mismos como una respuesta de afrontamiento ineficaz. Dentro del campo de investigación sobre regulación emocional, la rumiación también se ha considerado como una estrategia de afrontamiento de evitación (Smith y Alloy, 2009). Borkovec, Alcaine y Behar (2004) plantearon que otra forma de evitación cognitiva es la preocupación. Esta funcionaría como una evitación de amenazas percibidas. En este sentido la preocupación sobre cuestiones cotidianas puede cumplir dos funciones; una sería disminuir los pensamientos y sentimientos negativos sobre uno mismo o sobre intensas experiencias pasadas, y otra reducir la probabilidad subjetiva de sucesos negativos futuros mediante un mecanismo supersticioso, según el cual si uno se preocupa por algo, así evita que realmente se pueda producir. La preocupación, por tanto, estaría reforzada negativamente 
por la supresión inmediata de la reacción del sistema nervioso simpático ante material ansiógeno y por la no ocurrencia a largo plazo de los eventos temidos. Aunque estos investigadores han trabajado principalmente sobre el trastorno de ansiedad generalizada, plantean la relevancia de la preocupación en todos los trastornos emocionales. Finalmente, tanto los esfuerzos por desapegarse de las emociones negativas como el adoptar una perspectiva de tercera persona respecto a las mismas, se ha planteado que dificultan el procesamiento emocional. Este procesamiento emocional requiere la integración de los componentes cognitivos y afectivos de los recuerdos dolorosos (Foa y Kozak, 1986).

Otro concepto a destacar, especialmente en los trastornos de ansiedad, es el de evitación interoceptiva. Según Sandín (2005), ésta se produce debido al temor a los síntomas somáticos similares a los relacionados con la ansiedad (p.ej., incremento de la frecuencia cardíaca, etc.) que se producen de forma natural al realizar determinadas actividades (p.ej., correr, tomar café, etc.). La evitación interoceptiva suele ser sutil y en ella predomina la evitación a síntomas poco intensos. Así pues, es probable que la evitación interoceptiva se asocie a un grado elevado de sensibilidad a la ansiedad, pero no necesariamente a un nivel elevado de cogniciones catastrofistas (Sandín, 2005).

Por último, en el ámbito de la evitación experiencial, muchos trastornos mentales que aparecen en los sistemas de clasificación al uso podrían conceptualizarse como formas diversas de evitación no saludable (Hayes, Wilson, Gifford, Follette, y Strosahl, 1996). Dichos sistemas de clasificación diferencian los trastornos por los contenidos cognitivos, por los estímulos o circunstancias ante los que la gente reacciona y por la forma o el contenido de la respuesta. Pero en todos ellos es habitual que la persona se centre en el comportamiento de evitación experiencial. Así, Barlow et al. (2010) destacan la evitación emocional como un proceso central en muchas formas de psicopatología.

La evitación experiencial es perjudicial porque los eventos privados son paradójicamente incrementados por los esfuerzos deliberados de controlarlos (Hayes et al., 2004). El término evitación en este contexto incluye explícitamente evitación y escape en todas sus formas, teniendo en cuenta que ambos son métodos de alterar la forma o frecuencia de experiencias y los contextos que las ocasionan. De acuerdo con Butler y Ciarrochi (2007), la evitación es el polo contrario de la aceptación psicológica, siendo ambos parte del mismo constructo. Es de suponer que aquellas personas con un elevado comportamiento evitativo a su vez muestren un bajo nivel de aceptación psicológica, tanto de determinados déficits en su repertorio conductual o emocional, como de determinadas situaciones contextuales negativas o no satisfactorias y sobre las que cabe poco margen de modificación. En este sentido, cabe postular una relación inversa entre evitación y aceptación psicológica. Y también se plantea la implicación de ésta última en la psicopatología, considerando que una baja capacidad de aceptación puede ser un factor facilitador de la aparición de síntomas psicopatológicos varios, y por tanto de lo que se viene definiendo como trastornos mentales. Recientemente, incluso se está estudiando la aceptación psicológica de aspectos concretos y su posible implicación en trastornos específicos. Este sería el caso de la aceptación psicológica de la imagen corporal, la cual ha demostrado tener un efecto indirecto en la insatisfacción corporal presente en los trastornos de la conducta alimentaria (Sandoz, Wilson, Merwin y Kellum, 2013).

La presente investigación tiene como objetivo general comprobar si existen relaciones entre los componentes fundamentales de la evitación (cognitivo, conductual y experiencial), y la psicopatología de la ansiedad y la depresión. En este área de estudio quedan muchos interrogantes por resolver, especialmente en lo que se refiere al estudio comparativo de dichas variables entre población con y sin psicopatología, ya que son escasos los estudios experimentales desarrollados hasta ahora que las investiguen en muestras de personas con trastornos mentales. El objetivo fundamental de este trabajo es estudiar las citadas relaciones no sólo mediante medidas de autoinforme, sino también a través de una tarea experimental diseñada ad hoc. A continuación se describen las hipótesis de la presente investigación: 1) Existirán diferencias estadísticamente significativas en la tarea experimental de evitación entre la muestra clínica y el grupo control, siendo mayor la evitación en la muestra clínica. 2) Los sujetos con menores niveles de aceptación psicológica y de mindfulness, y con mayores niveles de evitación cognitivo-conductual, mostrarán mayor grado de evitación en la tarea experimental. 3) Los sujetos con mayores niveles de ansiedad y depresión mostrarán mayor grado de evitación en la tarea experimental.

\section{Método}

\section{Participantes}

Grupo clínico $(N=100)$. Pacientes con diagnóstico según criterios DSM-IV-TR (APA, 2001) de alguno de los siguientes trastornos. Subgrupo ansiedad $(n=50)$ : trastorno de angustia con o sin agorafobia, agorafobia sin historia de trastorno de angustia, fobia social, trastorno obsesivo-compulsivo, y trastorno de ansiedad ge- 
neralizada. Subgrupo depresión $(n=39)$ : trastorno depresivo mayor, trastorno distímico y trastorno depresivo no especificado. Y subgrupo trastorno límite de la personalidad (TLP) $(n=11)$, si bien éste no pertenece a las dos categorías anteriores, se ha incluido porque en estos sujetos la sintomatología ansiosa y depresiva ocurre de forma destacada. Todos los sujetos acudían a una consulta externa de Psicología Clínica en el Hospital Universitario de Guadalajara.

Grupo control $(N=100)$. Formado por personas de la población general sin antecedentes personales de trastornos mentales, que no estuvieran tomando psicofármacos y que no hubieran acudido a un psicólogo o psiquiatra en los últimos dos años por cualquier motivo. Estos sujetos fueron recabados a través de anuncios y a través del método de "bola de nieve" por el investigador y los participantes.

El rango de edad para los participantes fue 18-60 años. La edad media del grupo clínico fue de 35.39 años ( $D T=$ $10.74)$ y la del grupo control $36.21(D T=11.95)$. Sus características sociodemográficas aparecen en la Tabla 1.

Tabla 1. Características sociodemográficas

\begin{tabular}{llcc}
\hline \multirow{2}{*}{ Sexo } & Hombre & $\begin{array}{c}\text { Grupo clínico } \\
(n=100), \%\end{array}$ & $\begin{array}{c}\text { Grupo control } \\
(n=100), \%\end{array}$ \\
\hline Estado & Mujer & 42 & 29 \\
civil & Casado/vive en & 58 & 71 \\
& pareja & 54 & 51 \\
& Soltero & 40 & 39 \\
& Separado/ & 6 & 10 \\
& divorciado & & \\
\hline Estudios & EGB/ESO & 38 & 3 \\
& Bachillerato/FP & 41 & 41 \\
& Diplomatura & 12 & 27 \\
& Licenciatura & 9 & 29 \\
\hline Nivel & Bajo & 10 & 1 \\
económico & Medio-bajo & 69 & 56 \\
& Medio-alto & 21 & 43 \\
\hline Ocupación & Desempleado & 35 & 8 \\
& Estudiante & 15 & 24 \\
& Empleo público & 11 & \\
& Empleo privado & 39 & \\
\hline
\end{tabular}

\section{Procedimiento}

Se contó con la oportuna autorización del Comité Ético de Investigación Clínica del Hospital Universitario de Guadalajara. Todos los participantes firmaron un consentimiento informado antes de participar en el estudio. Tras la recogida de los datos sociodemográficos, se llevó a cabo la SCID-I (First, Spitzer, Gibbon y Williams, 1996) para establecer el diagnóstico adecuado en el caso de la muestra clínica y para descartar la presencia de cualquier trastorno mental en el caso de los sujetos del grupo control. La entrevista fue aplicada por el autor del trabajo, el cual cuenta con una amplia experiencia tanto en el ámbito clínico como en el uso de la misma. A continuación fueron aplicados los instrumentos de evaluación en el orden aleatorio que fue establecido. Los métodos de muestreo excluyeron el contrabalanceo, por tanto el orden de los instrumentos de evaluación fue constante. Finalmente los sujetos realizaron la tarea experimental de evitación. Todo el procedimiento fue realizado de forma individual.

\section{Instrumentos}

Entrevista Clínica Estructurada para los Trastornos del Eje I del DSM-IV (Structured Clinical Interview for DSM-IV Axis I Disorders, SCID-I; First et al., 1996). Es una entrevista semiestructurada. Se empleó la versión clínica (SCID-I-VC), que es una adaptación posterior perfeccionada para facilitar su utilización en la asistencia clínica. La decisión de incorporar la SCID-VC en esta investigación está basada en su amplia utilización en las publicaciones que quieren añadir cierto rigor al establecimiento de diagnósticos. Un gran número de estudios que utilizan la SCID centrándose en grupos diagnósticos particulares han obtenido valores kappa desde .70 hasta 1.00 (First et al., 1996).

\section{Medidas de autoinforme}

Escala de Conciencia y Atención Plena (Mindful Attention Awareness Scale, MAAS; Brown y Ryan, 2003). Consta de 15 ítems que se contestan mediante una escala tipo Likert de seis puntos. Su rango de puntuaciones es: 15-90. A mayor puntuación, mayor nivel de mindfulness. El nivel de consistencia interna informado por los autores originales fue satisfactorio $(\alpha=.82)$. Se ha optado por la MAAS debido a su brevedad, a su estructura unifactorial y a que es aplicable tanto a población clínica como general. Sauer et al. (2013), en un trabajo de revisión sobre los instrumentos de evaluación de mindfulness, concluyen que la MAAS es la escala más extendida. Estos autores también recomiendan su uso por su utilidad demostrada, especialmente en los casos en los que el investigador necesite una escala breve y esté interesado en una medida general de mindfulness más que en sus diversas facetas. Se empleó la adaptación española de Barajas y Garra (2014), la cual mostró un elevado nivel de consistencia interna $(\alpha=.88)$. 
Escala de Evitación Cognitivo-Conductual (Cognitive-Behavioral Avoidance Scale, CBAS; Ottenbreit y Dobson, 2004). Es una medida multidimensional de evitación, consta de cuatro subescalas: conductual social, conductual no social, cognitivo social y cognitivo no social. Tiene 31 ítems evaluados mediante una escala Likert de cinco puntos (rango: 31-155). A mayor puntuación mayor nivel de evitación. Ottenbreit y Dobson (2004) encontraron unas propiedades psicométricas satisfactorias tanto para la escala total $(\alpha=.91)$ como para sus subescalas (entre .75 y .86). En el presente estudio se ha utilizado la adaptación española de S. Barajas y L. Garra, con coeficientes alfa de Cronbach de .95 para la escala total, y entre .77 y .93 para las subescalas.

Cuestionario de Aceptación y Acción-II (Acceptance and Action Questionnaire-II, AAQ-II; Bond et al., 2011). Evalúa los constructos de aceptación, evitación experiencial e inflexibilidad psicológica. Consta de 10 ítems que se evalúan mediante una escala Likert de 7 puntos y donde los ítems 1, 6 y 10 son inversos. Por tanto, el rango oscila de 10-70. Puntuaciones más elevadas indicarían mayor evitación experiencial. Tiene un alfa de .87 (Bond et al., 2011). Aunque se ha considerado una versión final de siete ítems en la que los tres ítems inversos han sido eliminados, Ruiz, Langer, Luciano, Cangas y Beltrán (2013) resaltan que la versión de diez ítems ha sido ampliamente usada en estudios previos. También recuerdan, como ya hicieron Bond et al. (2011), que la versión de diez ítems no es significativamente más débil que la de siete y por tanto no debería asumirse que los estudios llevados a cabo con la primera sean inválidos.

Inventario de Depresión de Beck (Beck Depression Inventory-II, BDI-II; Beck, Steer y Brown, 1996). Es un instrumento ampliamente validado que evalúa la presencia de sintomatología depresiva y permite cuantificar su intensidad. Tiene 21 ítems con cuatro alternativas de respuesta. El rango de puntuaciones oscila entre 0 y 63 puntos. Tiene una elevada consistencia interna $(\alpha=.89)$. Se ha utilizado la versión española de Sanz, Navarro y Vázquez (2003).

Inventario de Ansiedad de Beck (Beck Anxiety Inventory, BAI; Beck y Steer, 1993). Sirve para evaluar la sintomatología ansiosa tanto en pacientes con trastornos psicológicos como en población normal. Tiene 21 ítems que se contestan mediante una escala Likert de 4 puntos, siendo el rango total de 0 a 63 . Se ha empleado la versión española de Sanz y Navarro (2003).

\section{Tarea experimental de evitación}

Ottenbreit y Dobson (2004) sugirieron que se desarrollara una tarea de evitación que pudiera compararse con las medidas de autoinforme de evitación. En este sentido se ha diseñado una tarea de evitación basada en la presentación de fotografías del IAPS (International Affective Picture System) (Lang, Bradley y Cuthbert, 2005). Las imágenes del IAPS han sido ampliamente utilizadas como estímulos emocionales (positivos, negativos o neutros) en estudios previos, especialmente en trastornos depresivos (Allen, Trinder y Brennan, 1999; Dichter, Tomarken, Shelton y Sutton, 2004; Dunn, Dalgleishl, Lawrence, Cusack y Ogilvie, 2004; Forbes, Miller, Gohn, Fox y Kovacs, 2005; Sloan, Strauss, Quirk y Sajatovic, 1997; Sloan, Strauss y Wisner, 2001).

El IAPS es un conjunto estandarizado de fotografías fiable y válido para el estudio experimental de las emociones, construido a partir de la aproximación dimensional de las emociones propuesta por Peter J. Lang. Este autor y su equipo plantearon la existencia de dos dimensiones bipolares en la estructura afectiva: la valencia afectiva (que se mueve en el rango agradable-desagradable) y el arousal o activación fisiológica (cuyo rango iría de la excitación a la calma). También postularon, de manera menos consistente y con un peso mucho menor, una tercera dimensión llamada dominancia o control que también encontraron correlacionada con la dimensión de valencia (Lang et al., 2005). El IAPS cuenta con estímulos objetivos, controlables y cuyos efectos sobre cada uno de los tres componentes de la respuesta afectiva son conocidos. Su pretensión metodológica es posibilitar estímulos emocionales calibrados que puedan servir como una medida estandarizada.

El IAPS ha sido adaptado a población española por Moltó et al. (1999) y Vila et al. (2001). Dichos autores al comparar sus datos con los de las estimaciones estadounidenses encontraron una elevada correlación, sugiriendo que los estímulos del IAPS son entendidos de la misma manera por españoles y norteamericanos, salvando así factores culturales y geográficos.

Para el presente trabajo se seleccionaron sesenta imágenes de las más de 800 del IAPS validadas en población española. Veinte de ellas tenían una valencia afectiva agradable, veinte desagradable y otras veinte neutra. En las tres dimensiones el valor de las fotografías oscila en un rango de 1 a 9, donde valores bajos indicarían baja valencia afectiva (desagradables), baja activación (elicitada) y baja dominancia (bajo control). Puede comprobarse un listado de dichas fotografías en el Anexo 1 con su puntuación en las tres dimensiones, así como los valores medios para cada uno de los tres grupos establecidos en función de la valencia afectiva. Los sujetos debían anotar en un folio el número de cada fotografía que querían evitar volver a ver. Esta acción debían realizarla cada vez que apareciera una imagen con dicho criterio y para ello tenían que soltar el ratón con el que controla- 
ban su paso, coger el bolígrafo y anotar los cuatro dígitos indicativos de la fotografía en cuestión. Dichos dígitos aparecían en pequeño tamaño, de tal forma que se requería un esfuerzo atencional para visualizarlos y acto seguido anotarlos, quedando así clara la naturaleza cognitiva y conductual de esta tarea. No se informaba previamente sobre las características de las imágenes, ni el número total de ellas, pudiendo anotar tantas como estimaran oportunas. Inicialmente se presentaban dos imágenes de prueba, de carácter neutro.

En la Tabla 2 puede verse la distribución de las sesenta fotografías seleccionadas, teniendo en cuenta que la dimensión relevante para este estudio era la valencia afectiva. Es decir, obtener estímulos objetivos de carácter desagradable, neutro y agradable. Al igual que en el conjunto original de más de 800 fotografías validadas por Moltó et al. (1999) y Vila et al. (2001), resulta casi imposible encontrar estímulos desagradables que produzcan poca activación. Se considera que el criterio de selección empleado es bastante estricto, por ejemplo en comparación con criterios de otros investigadores como Sánchez-García, Martínez-Sánchez y Van der Hofstadt (2011) que establecieron los siguientes criterios: valencia alta (puntuación > 6), media (puntuación 4-6) y baja $(<3.5)$.

Tabla 2. Distribución del número de imágenes seleccionadas del IAPS en función de su puntuación en valencia afectiva y activación

\begin{tabular}{lccc}
\hline \multirow{2}{*}{ Activación } & \multicolumn{3}{c}{ Valencia afectiva } \\
\cline { 2 - 4 } & $\begin{array}{c}\text { Baja }(<2.75) \\
(\text { desagradables) }\end{array}$ & $\begin{array}{c}\text { Media (5-5.5) } \\
\text { (neutras) }\end{array}$ & $\begin{array}{c}\text { Alta }(>7.5) \\
\text { (agradables) }\end{array}$ \\
\hline Baja $(<5)$ & 2 & 10 & 8 \\
Media (5-5.5) & 1 & 5 & 1 \\
Alta $(>5.5)$ & 17 & 5 & 11 \\
\hline
\end{tabular}

\section{Medicación}

En el grupo clínico se analizó la relación entre la toma de psicofármacos y el resto de variables estudiadas. La prescripción de dicha medicación no estaba controlada por el investigador. En todos los casos los pacientes la llevaban tomando un periodo mínimo de un mes. En los casos de pacientes que llevaban tomando psicofármacos durante periodos prolongados, incluso varios años, la medicación que se ha tenido en cuenta es la que tomaban en el último mes, independientemente de que en otros periodos hubieran tomado más o menos psicofármacos, iguales o diferentes. Dadas las múltiples combinaciones resultantes en el tipo de psicofármacos que estaban tomando los sujetos clínicos de la muestra (ansiolíticos, antidepresivos, antipsicóticos y fármacos de la familia de los antiepilépticos) y que éstas a veces no respondían a la supuesta lógica que debería conectar el tipo de trastorno padecido con el tipo de medicación recibida (ej.: un paciente con un trastorno de ansiedad podía estar recibiendo un fármaco antidepresivo, un paciente con un trastorno depresivo podía estar recibiendo un fármaco ansiolítico, un paciente con un TLP podía estar recibiendo cualquiera de los dos anteriores o uno antiepiléptico o los tres simultáneamente, etc.), se decidió tener en cuenta exclusivamente la toma o no de medicación. En caso de haberse tenido en cuenta cada tipo de psicofármaco y su posible combinación con el resto, más la categoría diagnóstica a la que pertenecía el paciente, la casuística resultante habría supuesto comparar numerosos grupos con escasos sujetos en cada uno.

\section{Análisis estadísticos}

Para la realización de los análisis estadísticos se empleó la versión 17 del programa SPSS. Como pudo comprobarse en la Tabla 1, los grupos difieren de forma importante y estadísticamente significativa en las variables: nivel de estudios, nivel socioeconómico y ocupación, por lo que podrían resultar potenciales factores de confusión. Para comprobarlo se realizaron los siguientes análisis: Rho de Spearman para la variable edad, U de Mann-Whitney más W de Wilcoxon para la variable sexo, y Chi-cuadrado más prueba de Kruskal-Wallis para las variables estado civil, estudios, nivel socioeconómico y ocupación. Se comprobó que en el conjunto de los grupos, el nivel de estudios, el nivel socioeconómico y la ocupación eran potenciales factores de confusión. Por este motivo en el siguiente apartado se ofrecen los valores de significación ajustados por dichos factores de confusión. Las relaciones entre la tarea de evitación y el resto de medidas de autoinforme fueron estudiadas mediante el coeficiente de correlación de Spearman (Rho de Spearman).

\section{Resultados}

En primer lugar se comprobó que los tres grupos de fotografías establecidos diferían significativamente en la dimensión valencia y que no diferían en la dimensión arousal, siendo irrelevante en este caso la dimensión dominancia. En la Tabla 3 aparecen las medias de valencia, arousal y dominancia según el tipo de fotografía. Como puede comprobarse, las de valencia son muy diferentes, las de dominancia son también diferentes pero en menor medida y las de arousal son similares. Para comprobar que dichas diferencias eran significativas se llevó a cabo un análisis de varianza univariante (ANOVA). Las medias de valencia y dominancia no son iguales (valencia: 
$F=3294.43$, g.l. $=2, p<.001$; dominancia: $F=98.11$, g.l. $=2, p<.001)$, mientras que las medias de arousal sí lo son $(F=1.31$, g.l. $=2, p=.28)$. En un segundo paso, se realizó una prueba de comparaciones múltiples con la corrección de Bonferroni, para asegurar que todas las diferencias en valencia y dominancia diferían dos a dos ( $p$ $<.001)$, mientras que no existía tal diferencia en arousal (ver Tabla 3). Para llegar a estos análisis estadísticos satisfactorios fue necesario hacer varias combinaciones previas de distintas selecciones de imágenes hasta dar con aquella que cumpliera los criterios necesarios.

Tabla 3. Prueba de comparaciones múltiples con corrección de Bonferroni

\begin{tabular}{|c|c|c|c|c|c|}
\hline & Media & $D T$ & Tipo foto (A) & Tipo foto (B) & $(\mathrm{A})-(\mathrm{B})$ \\
\hline \multirow{9}{*}{$\begin{array}{l}\mathrm{V} \\
\mathrm{A} \\
\mathrm{L} \\
\mathrm{E} \\
\mathrm{N} \\
\mathrm{C} \\
\mathrm{I} \\
\mathrm{A}\end{array}$} & \multirow[t]{3}{*}{7.84} & \multirow[t]{3}{*}{0.20} & \multirow[t]{3}{*}{ Agradable } & Agradable & \\
\hline & & & & Neutra & $2.59 * * *$ \\
\hline & & & & Desagradable & $5.49 * * *$ \\
\hline & \multirow[t]{3}{*}{5.25} & \multirow[t]{3}{*}{0.17} & \multirow[t]{3}{*}{ Neutra } & Agradable & $-2.59 * * *$ \\
\hline & & & & Neutra & \\
\hline & & & & Desagradable & $2.90 * * *$ \\
\hline & \multirow[t]{3}{*}{2.35} & \multirow[t]{3}{*}{0.26} & \multirow[t]{3}{*}{ Desagradable } & Agradable & $-5.49 * * *$ \\
\hline & & & & Neutra & $-2.90 * * *$ \\
\hline & & & & Desagradable & \\
\hline \multirow{9}{*}{$\begin{array}{l}\mathrm{A} \\
\mathrm{R} \\
\mathrm{O} \\
\mathrm{U} \\
\mathrm{S} \\
\mathrm{A} \\
\mathrm{L}\end{array}$} & \multirow[t]{3}{*}{5.32} & \multirow[t]{3}{*}{1.04} & \multirow[t]{3}{*}{ Agradable } & Agradable & \\
\hline & & & & Neutra & $0.10 ;$ n.s. \\
\hline & & & & Desagradable & -0.32 ; n.s. \\
\hline & \multirow[t]{3}{*}{5.22} & \multirow[t]{3}{*}{0.92} & \multirow[t]{3}{*}{ Neutra } & Agradable & -0.10 ; n.s. \\
\hline & & & & Neutra & \\
\hline & & & & Desagradable & $-0.42 ;$ n.s. \\
\hline & \multirow[t]{3}{*}{5.64} & \multirow[t]{3}{*}{0.64} & \multirow[t]{3}{*}{ Desagradable } & Agradable & 0.32 ; n.s. \\
\hline & & & & Neutra & $0.42 ;$ n.s. \\
\hline & & & & Desagradable & \\
\hline $\mathrm{D}$ & \multirow[t]{3}{*}{5.97} & \multirow[t]{3}{*}{0.62} & \multirow[t]{3}{*}{ Agradable } & Agradable & \\
\hline $\mathrm{O}$ & & & & Neutra & $1.04 * * *$ \\
\hline M & & & & Desagradable & $2.52 * * *$ \\
\hline $\begin{array}{c}\mathrm{I} \\
\mathrm{N}\end{array}$ & \multirow[t]{3}{*}{4.93} & \multirow[t]{3}{*}{0.61} & \multirow[t]{3}{*}{ Neutra } & Agradable & $-1.04 * * *$ \\
\hline $\mathrm{N}$ & & & & Neutra & \\
\hline $\begin{array}{l}\mathrm{A} \\
\mathrm{N}\end{array}$ & & & & Desagradable & $1.48 * * *$ \\
\hline $\mathrm{C}$ & \multirow[t]{3}{*}{3.45} & \multirow[t]{3}{*}{0.48} & \multirow[t]{3}{*}{ Desagradable } & Agradable & $-2.52 * * *$ \\
\hline I & & & & Neutra & $-1.48 * * *$ \\
\hline A & & & & Desagradable & \\
\hline
\end{tabular}

Nota: $(\mathrm{A})-(\mathrm{B})=$ diferencia de medias. $\mathrm{n} . \mathrm{s} .=$ no significativo. $* * * p<.001$

La variable dependiente fue el número de fotografías desagradables que los sujetos evitaban volver a visualizar. Esta variable obtuvo valores cero en cinco sujetos del grupo control y solamente en uno del grupo clínico. Aunque esta circunstancia ocurriera en total en pocos sujetos, implicaba que los datos no se ajustaran a una dis- tribución gamma. Por ello, se recodificó categorizándola a quintiles y se ajustó a un modelo de regresión logística ordinal multinomial (MLG). Se llevó a cabo un ajuste por edad, sexo, ocupación y nivel socioeconómico. Los dos grupos difirieron significativamente en esta variable, mostrando el grupo clínico un mayor nivel de evitación que el grupo control $(p<.001)$. El grupo clínico obtuvo una media de $12.74(D T=5.76)$ imágenes desagradables evitadas, frente al grupo control que indicó una media de $9.08(D T=5.84)$. No aparecieron diferencias significativas $(p=.62)$ entre los tres subgrupos clínicos (subgrupo ansiedad $=12.44, D T=6.31$; subgrupo depresión $=$ $13.28, D T=4.95$; subgrupo TLP $=12.18, D T=6.18)$. No existieron diferencias de género (Grupo clínico: varones $=12.48, D T=5.57$; mujeres $=12.93, D T=5.93 ; p=.72$. Grupo control: varones $=9.07, D T=5.81 ;$ mujeres $=$ $9.08, D T=5.90 ; p=.79)$. Tampoco se observó correlación destacable entre las fotografías desagradables evitadas y la edad de los sujetos (Grupo clínico: $r h o=.279, p$ $=.005$, aunque $p$ fue significativo la intensidad de la correlación es débil. Grupo control: rho $=.033, p=.743$ ).

No se encontró asociación estadísticamente significativa en ninguno de los dos grupos entre el nivel de evitación y el nivel de mindfulness (Grupo clínico: rho $=-.132, p=.189$. Grupo control: $r h o=.051, p=.616$ ). Es decir, no hubo asociación entre el número de estímulos visuales desagradables evitados y la puntuación en la MAAS. Tampoco se encontró relación entre el nivel de evitación en la tarea experimental y la puntuación total de la escala de evitación cognitivo-conductual (CBAS) (Grupo clínico: $r h o=.098, p=.33$. Grupo control: $r h o=$ $-.031, p=.761)$. Respecto a la relación entre la tarea de evitación y la evitación experiencial medida a través del AAQ-II, en el grupo clínico no existió correlación significativa entre ambas $(r h o=.039 ; p=.701)$. Mientras que en el grupo control, si bien apareció una correlación significativa, esta fue débil ( $r h o=.219 ; p=.029)$.

El nivel de evitación en la tarea experimental correlacionó significativamente en ambos grupos con la sintomatología ansioso-depresiva. Así, en el caso de la relación con depresión (BDI-II), se obtuvo una correlación (rho) de $223(p=.025)$ en el grupo clínico y de 246 ( $p$ $=.014)$ en el grupo control. Y en el caso de la relación con ansiedad (BAI), se obtuvo una correlación (rho) de $.241(p=.016)$ en el grupo clínico y de $.219(p=.029)$ en el grupo control. Aunque estas correlaciones fueron significativas, su intensidad fue débil.

Respecto al uso de medicación en el grupo clínico, la mayoría de los pacientes estaba medicado (58\%). El tipo de psicofármaco más usado fueron los antidepresivos (53\%). El subgrupo de trastornos de ansiedad era el menos medicado (56\% no tomaban medicación), seguido 
del de trastornos depresivos (30.77\%) y del de TLP $(18.18 \%)$. Los varones se encontraban más medicados (61.91\%) que las mujeres (55.18\%). Por último, era más frecuente el uso simultáneo de varios tipos de psicofármacos (33\%) que el uso de uno solo (25\%). Se analizó si había diferencias entre los pacientes medicados y los no medicados, en las medidas de autoinforme y en la tarea de evitación, encontrándose diferencias significativas en la puntuación de la MAAS y la CBAS. De tal forma que los pacientes medicados presentaban un menor nivel de mindfulness (MAAS: $M=3.42, D T=0.77$ ) que los no medicados (MAAS: $M=3.77, D T=0.74)$ (MLG, logística ordinal multinomial: $p=.016$ ). Además, los pacientes medicados mostraban un mayor nivel de evitación (CBAS Total: $M=86.05, D T=23.02$ ) que los no medicados (CBAS Total: $M=75.81, D T=19.68)(p=.028)$. Los valores de $p$ se ajustaron por grupo diagnóstico. Aunque los pacientes medicados, en conjunto, obtuvieron también puntuaciones más elevadas en el BDI-II, BAI, AAQ-II y en la tarea experimental de evitación que los no medicados, estas diferencias no resultaron estadísticamente significativas.

\section{Discusión}

En la presente investigación se ha confirmado que existen diferencias estadísticamente significativas en la tarea experimental de evitación entre la muestra clínica y la muestra control, siendo mayor el grado de evitación en la muestra clínica. Es decir, los sujetos con sintomatología clínica de ansiedad y depresión evitan en mayor medida volver a ver estímulos visuales de valencia afectiva desagradable que los sujetos sin psicopatología. Se puede afirmar pues que la tarea de evitación diseñada posee validez discriminante. Aunque también se ha demostrado la existencia de una relación entre la sintomatología de ansiedad/depresión y el nivel de evitación exhibido en la tarea, hay que destacar que la intensidad de dichas relaciones es débil tanto en el grupo clínico como en el grupo control. Por este motivo serían precisos estudios futuros, tanto con población general como clínica, que confirmen esta asociación.

Los resultados obtenidos con este sencillo procedimiento experimental suponen un aval empírico para los planteamientos teóricos sobre la importancia de la evitación en la psicopatología (Barlow, 2002; Barlow, Allen y Choate, 2004). Además apoyan la relevancia que la evitación juega no sólo en los trastornos de ansiedad, en los cuales sí había sido considerada tradicionalmente, sino también en la depresión, como más recientemente se ha postulado en la Activación Conductual (Jacobson et al., 2001; Kanter et al., 2011; Martell et al., 2001;
Martell et al., 2013). Igualmente, apoyan la idea planteada al inicio de este trabajo de que en cualquier persona con un trastorno mental podría identificarse alguna forma relevante de evitación y que por tanto la evitación estaría de un modo u otro en la base de dichos trastornos mentales. De estos hallazgos se derivan implicaciones clínicas, puesto que uno de los objetivos fundamentales en los tratamientos psicológicos debería ser la identificación de las distintas modalidades y conductas de evitación en cada paciente y su posterior reducción, fomentando el contacto (exposición) con los estímulos y contextos asociados. En este sentido sería de interés en investigaciones futuras estudiar la ejecución de esta tarea en sujetos clínicos en relación con la evolución de su sintomatología. Por ejemplo, ver si se producen cambios en dicha ejecución en relación a la mejoría producida por un tratamiento.

No aparecen diferencias de género en la ejecución de la tarea experimental de evitación. En este sentido se hace necesaria una mayor investigación, ya que Blalock y Joiner (2000) sí informaron de un uso diferencial de estrategias de evitación entre varones y mujeres, y postularon que ambos desarrollarían estilos de afrontamiento diferentes. Por otra parte, la edad de los sujetos no resulta ser una variable relevante en el grado de evitación.

En contra de lo esperado no se encontraron relaciones significativas entre la tarea experimental de evitación y las escalas de mindfulness y de evitación cognitivo-conductual. Hay que tener en cuenta que hasta ahora no todos los estudios que han investigado las relaciones entre mindfulness y evitación mediante algún tipo de tarea experimental, en vez de a través de autoinforme, han encontrado resultados consistentes. En este sentido, Arch y Craske (2006) en un estudio con estudiantes divididos en tres condiciones experimentales (inducción de estado de preocupación, inducción de atención sin focalizar e inducción de estado mindfulness mediante focalización en la respiración) en el que podían elegir ver o no una serie de fotografías de valencia negativa del IAPS (Lang et al., 2005), encontraron que los participantes de la condición 'respiración focalizada' elegían ver más fotografías negativas que los de la condición 'atención no focalizada'. Pero cuando compararon el grupo 'respiración focalizada' con el de 'preocupación' no encontraron diferencias significativas. Sin embargo, mediante autoinforme, el grupo de 'respiración focalizada' sí informó significativamente de más estabilidad emocional durante la tarea que los otros dos grupos. Especialmente que el de 'preocupación'. Por tanto, son necesarias más investigaciones que analicen las relaciones entre evitación y mindfulness mediante métodos alternativos a los autoinformes y que se lleven a cabo tanto con muestras clínicas como no clí- 
nicas. Entre dichos métodos alternativos podría señalarse la tarea de evitación aquí diseñada, y en el caso de evaluación de mindfulness, algunos de los métodos sugeridos por Sauer et al. (2013) tales como medidas basadas en lenguaje, evaluación por evaluadores externos o medidas neuropsicológicas y biológicas.

Cabe preguntarse por qué sí aparece relación entre la tarea de evitación y la evitación experiencial en el grupo control pero no así en el grupo clínico. Una posible explicación al respecto podría ser que los sujetos con sintomatología psicopatológica de ansiedad y depresión sean muy evitativos de los eventos internos, pero no así de estímulos externos que aunque sean desagradables probablemente no estén relacionados con dichos eventos internos o experiencias personales. Algunos estudios apoyan también la existencia de relación entre evitación experiencial y algún tipo de tarea experimental de evitación, pero han sido llevados a cabo solamente con muestras no clínicas. Por ejemplo, Eifert y Heffner (2003) expusieron a una estimulación provocadora de ansiedad (inhalación de aire enriquecido en dióxido de carbono durante diez minutos) a sesenta mujeres estudiantes con elevada sensibilidad a la ansiedad, las cuales habían sido distribuidas en tres condiciones experimentales: contexto de aceptación, contexto de control (respiración abdominal) y condición de no instrucciones. Estos autores comprobaron que aquellas mujeres asignadas a la condición de 'aceptación' empezaban significativamente antes la exposición al $\mathrm{CO}_{2}$ (lo cual se consideró indicativo de menor evitación) e informaban de menos síntomas de miedo y de menor intensidad, que las de la condición de 'control'. Aunque los grupos no se diferenciaron en cuanto a las respuestas fisiológicas ni a su intensidad. Por tanto, se precisan más estudios con muestras clínicas para corroborar los resultados aquí encontrados.

Por otra parte, ya que se ha comprobado que los pacientes medicados con algún psicofármaco tienen mayores niveles de evitación cognitivo-conductual y menores niveles de mindfulness que los no medicados, es preciso conocer si esta diferencia significativa se debe a que los medicados tienen mayor psicopatología, o a que la medicación influye negativamente en dichas variables psicológicas. Dada la imposibilidad de contestar a esta pregunta en la presente investigación, puesto que la metodología empleada es correlacional, y dadas las importantes implicaciones clínicas que podrían derivarse, sería necesario en investigaciones futuras explorar posibles relaciones de causa-efecto entre dichas variables.

Para finalizar se señalan algunas limitaciones. Este estudio comprende una tarea de evitación diseñada ad hoc y sobre la cual por tanto no hay datos previos. Por esta razón, solamente estudios posteriores que generali- cen su utilización podrán determinar su verdadera funcionalidad. Tampoco se ha podido contar con un software de presentación de estímulos que permitiera, por ejemplo, medir otros datos relevantes como la latencia de respuesta, lo cual sería aconsejable en un uso futuro.

\section{Referencias}

Allen, N. B., Trinder, J. y Brennan, C. (1999). Affective startle modulation in clinical depression: Preliminary findings. Biological Psychiatry, 46, 542-550.

American Psychiatric Association. (2001). Manual diagnóstico y estadístico de los trastornos mentales. DSM-IV-TR. Barcelona: Masson.

Arch, J. J. y Craske, M. G. (2006). Mechanisms of mindfulness: Emotion regulation following a focused breathing induction. Behaviour Research and Therapy, 44, 1849-1858.

Barajas, S. y Garra, L. (2014). Mindfulness and psychopathology: Adaptation of the Mindful Attention Awareness Scale (MAAS) in a Spanish sample. Clínica y Salud, 25, 49-56.

Barlow, D. H. (2002). Anxiety and its disorders: the nature and treatment of anxiety and panic. New York: Guilford Press.

Barlow, D. H., Allen L.B. y Choate, M.L. (2004).Toward a unified treatment for emotional disorders. Behavior Therapy, 35 , 205-230.

Barlow, D. H., Farchione, T. J., Fairholme, C. P., Ellard, K. K., Boisseau, C. L., Allen, L. B., et al. (2010). Unified protocol for transdiagnostic treatment of emotional disorders: Therapist guide (1st ed.). USA: Oxford University Press.

Blalock, J. A. y Joiner, Jr., T. E. (2000). Interaction of cognitive avoidance coping and stress in predicting depression/anxiety. Cognitive Therapy and Research, 24, 47-65.

Beck, A. T., y Steer, R. A. (1993). Beck Anxiety Inventory. Manual. San Antonio, TX: The Psychological Corporation.

Beck, A. T., Steer, R. A., y Brown, G. K. (1996). BDI-II. Beck Depression Inventory-Second Edition. Manual. San Antonio, TX: The Psychological Corporation.

Bond, F. W., Hayes, S. C., Baer, R. A., Carpenter, K., Guenole, N., Orcutt, H. K., et al. (2011). Preliminary psychometric properties of Acceptance and Action Questionnaire-II. A revised measure of psychological inflexibility and experiential avoidance. Behavior Therapy, 42, 676-688.

Borkovec, T. D., Alcaine, O., y Behar, E. (2004). Avoidance theory of worry and generalized anxiety disorder. En R.G. Heimberg, C.L. Turk, y D.S. Mennin (Eds.) Generalized anxiety disorder: Advances in research and practice (pp.77108). New York: Guilford Press.

Brown, K. W. y Ryan, R. M. (2003). The benefits of being present: mindfulness and its role in psychological well-being. Journal of Personality and Social Psychology, 84, 822-848.

Butler, J. y Ciarrochi, J. (2007). Psychological acceptance and quality of life in the elderly. Quality of Life Research, 16, 607-615.

Campos, M., Iraurgui, J., Páez, D. y Velasco, C. (2004). Afrontamiento y regulación emocional de hechos estresantes un meta-análisis de 13 estudios. Boletín de Psicología, 82, 25-44.

Dichter, G., Tomarken, A., Shelton, R. y Sutton, S. (2004). Early and late onset startle modulation in unipolar depression. Psychophysiology, 41, 433-440. 
Dunn, B. D., Dalgleishl, T., Lawrence, A. D., Cusack, R. y Ogilvie, A. (2004). Categorical and dimensional reports of experienced affect to emotion-inducing pictures in depression. Journal of Abnormal Psychology, 113, 654-660.

Eifert, G. H. y Heffner, M. (2003). The effects of acceptance versus control contexts on avoidance of panic-related symptoms. Journal of Behavior Therapy and Experimental Psychiatry, 34, 293-312.

First, M. B., Spitzer, R. L., Gibbon, M. y Williams, J. B. (1996). Structured Clinical Interview for DSM-IV Axis I Disorders, Clinician Version ( $S C I D-C V$ ). Washington, D.C.: American Psychiatric Press. (Existe traducción al castellano como Entrevista clínica estructurada para los trastornos del eje I del DSM-IV, Versión clínica, Guía del Usuario. 1999. Barcelona: Masson).

Foa, E. y Kozak, M. J. (1986). Emotional processing of fear: exposure to corrective information. Psychological Bulletin, 99, 20-35.

Forbes, E. E., Miller, A., Gohn, J., Fox, N. y Kovacs, M. (2005). Affect-modulated startle in adults with childhood onset depression: relations to bipolar course and number of lifetime depressive episodes. Psychiatry Research, 134, 11-25.

Hayes, S. C., Strosahl, K. D., Wilson, K. G., Bissett, R. T., Pistorello, J., Toarmino, D., et al. (2004). Measuring experiential avoidance: a preliminary test of a working model. The Psychological Record, 54, 553-578.

Hayes, S. C., Wilson, K. G., Gifford, E.V., Follette, V. M. y Strosahl, K. D. (1996). Experiential avoidance and behavioral disorders: A functional dimension approach to diagnosis and treatment. Journal of Consulting and Clinical Psychology, 64, 1152-1168.

Hernangómez, L. y Vázquez, C. (2005). Regulatory strategies for depressed mood: Differences between patients and non-patients in the Coping Inventory of Depression (CID). EduPsykhe: Revista de Psicología y Psicopedagogía, 4, 149-178.

Holahan, C. J., Moos, R.H, Holahan, C. K., Brennan, P. L. y Schutte, K. K. (2005). Stress generation, avoidance coping and depressive symptoms: a 10-year model. Journal of Consulting and Clinical Psychology, 73, 658-666.

Jacobson, N. S., Martell, C. R. y Dimidjian, S. (2001). Behavioral activation treatment for depression: returning to contextual roots. Clinical Psychology: Science and Practice, 8, 225-270.

Kanter, J. W., Busch, A. M. y Rusch, L. C. (2011). Activación conductual: refuerzos positivos ante la depresión. Madrid: Alianza Editorial. (Obra original publicada en 2009).

Kashdan, T. B., Barrios, V., Forsyth, J. P. y Steger, M. F. (2006). Experiential avoidance as a generalized psychological vulnerability: comparisons with coping and emotion regulation strategies. Behaviour Research and Therapy, 44, 1301-1320.

Lang P. J., Bradley M. M. y Cuthbert B. N. (2005). International affective picture system (IAPS): Affective ratings of pictures and instruction manual. Technical Report A-6. University of Florida, Gainesville, FL. (Obtenido a través de: http://csea. phhp.ufl.edu/Media.html).

Martell, C. R., Addis, M. E. y Jacobson, N.S. (2001). Depression in context: Strategies for guided action. New York: W. W. Norton.

Martell, C. R., Dimidjian, S. y Herman-Dunn, R. (2013). Activación conductual para la depresión: una guía clínica. Bilbao: Desclée de Brouwer. (Obra original publicada en 2010).

Moltó, J., Montañes, S., Poy, R., Segarra, P., Pastor, C., Tormo, P., et al. (1999). Un nuevo método para el estudio experimental de las emociones: el International Affective Picture System
(IAPS). Adaptación española. Revista de Psicología General y Aplicada, 52, 55-87.

Morris, E. M. J., Johns, L. C. y Oliver, J. E. (Eds.) (2013). Acceptance and commitment therapy and Mindfulness for psychosis. Chichester, England: Wiley-Blackwell.

Ottenbreit, N. D. y Dobson, K. S. (2004). Avoidance and depression: the construction of the Cognitive-Behavioral Avoidance Scale. Behaviour Research and Therapy, 42, 292-313.

Ottaviani, C., Borlimi, R., Brighetti, G., Caselli, G., Favaretto, E., Giardini, I., et al. (2014). Worry as an adaptive avoidance strategy in healthy controls but not in pathological worriers. International Journal of Psychophysiology, 93, 349-355.

Pérez-Álvarez, M., García-Montes, J. M., Perona-Garcelán, S. y Vallina-Fernández, O. (2008). Changing Relationship with Voices: New Therapeutic Perspectives for Treating Hallucinations. Clinical Psychology and Psychotherapy, 15, 75-85.

Ruiz, F. J., Langer, A. I., Luciano, C., Cangas, A. J. y Beltrán, I. (2013). Measuring experiential avoidance and psychological inflexibility: the Spanish version of the Acceptance and Action Questionnaire-II. Psicothema, 25, 123-129.

Sánchez-García, M., Martínez-Sánchez, F. y Van der Hofstadt, C. (2011). Alexitima y reconocimiento de emociones inducidas experimentalmente en personas con somatizaciones. Psicothema, 23, 707-712.

Sandín, B. (2005). Evitación interoceptiva: nuevo constructo en el campo de los trastornos de ansiedad. Revista de Psicopatología y Psicología Clínica, 10, 103-114.

Sandoz, E. K., Wilson, K. G., Merwin, R. M, y Kellum, K. K. (2013). Assessment of body image flexibility: The Body Image-Acceptance and Action Questionnaire. Journal of Contextual Behavioral Science, 2, 39-48.

Sanz, J. y Navarro, M. E. (2003). Propiedades psicométricas de una versión española del Inventario de Ansiedad de Beck (BAI) en estudiantes universitarios. Ansiedad y Estrés, 9, 59-84.

Sanz, J., Navarro, M. E. y Vázquez, C. (2003). Adaptación española del Inventario para la Depresión de Beck-II (BDI-II). Propiedades psicométricas en estudiantes universitarios. Análisis y Modificación de Conducta, 29, 239-288.

Sauer, S., Walach, H., Schmidt, S., Hinterberger, T., Lynch, S., Bussing, A. y Kohls, N. (2013). Assessment of mindfulness: Review on state of the art. Mindfulness, 4, 3-17.

Sloan, D. M., Strauss, M. E., Quirk, S. W. y Sajatovic, M. (1997). Subjective and expressive emotional responses in depression. Journal of Affective Disorders, 46, 135-141.

Sloan, D. M., Strauss, M. E. y Wisner, K. L. (2001). Diminished response to pleasant stimuli by depressed women. Journal of Abnormal Psychology, 110, 488-493.

Smith, J. M. y Alloy, L. B. (2009). A roadmap to rumination: a review of the definition, assessment, and conceptualization of this multifaceted construct. Clinical Psychology Review, 29, 116-128.

Vila, J., Sánchez, M., Ramírez, I., Fernández, C., Cobos, P., Rodríguez, S., et al. (2001). El sistema internacional de imágenes afectivas (IAPS): Adaptación española. Segunda parte. Revista de Psicología General y Aplicada, 54, 635-657.

Vilardaga, R., Hayes, S. C., Atkins, D. C., Bresee, C. y Kambiz, A. (2013). Comparing experiential acceptance and cognitive reappraisal as predictors of functional outcome in individuals with serious mental illness. Behaviour Research and Therapy, $51,425-433$. 
Anexo 1. Fotos seleccionadas del IAPS

\begin{tabular}{|c|c|c|c|c|}
\hline FOTO N & DESCRIPCIÓN & $\mathrm{V}^{*}$ & $A^{*}$ & $\mathrm{D}^{*}$ \\
\hline \multicolumn{5}{|c|}{ AGRADABLES } \\
\hline 1340 & mujeres & 7.96 & 4.29 & 6.25 \\
\hline 2165 & padre & 8.19 & 4.98 & 6.66 \\
\hline 2341 & niños & 8.1 & 4.55 & 6.39 \\
\hline 2352.1 & beso & 7.8 & 4.96 & 6.14 \\
\hline 2395 & familia & 7.62 & 4.04 & 5.83 \\
\hline 4599 & pareja & 7.93 & 5.68 & 6.12 \\
\hline 5201 & cerezos & 7.76 & 3.2 & 5.71 \\
\hline 5260 & cataratas & 7.82 & 5.88 & 4.3 \\
\hline 5470 & astronauta & 7.57 & 5.63 & 5.38 \\
\hline 5621 & paracaidistas & 7.61 & 6.92 & 5.78 \\
\hline 5628 & pico montaña & 7.8 & 6.11 & 6.87 \\
\hline 5629 & excursionista & 7.51 & 6.31 & 5.31 \\
\hline 5760 & paisaje & 8.1 & 3.96 & 5.8 \\
\hline 5831 & gaviotas & 8.03 & 4.34 & 6.22 \\
\hline 7220 & pasteles & 7.58 & 5.51 & 6.58 \\
\hline 7330 & helado & 7.87 & 5.27 & 6.2 \\
\hline 7502 & castillo & 8.06 & 5.52 & 5.68 \\
\hline 8380 & atletas & 7.88 & 5.84 & 6.79 \\
\hline 8461 & adolescentes & 7.91 & 6.02 & 6.28 \\
\hline 8490 & montaña rusa & 7.78 & 7.39 & 5.16 \\
\hline media & & 7.84 & 5.32 & 5.97 \\
\hline \multicolumn{5}{|l|}{ NEUTRAS } \\
\hline 1313 & rana & 5.05 & 4.99 & 4.96 \\
\hline 1726 & tigre & 5.16 & 7.3 & 3.41 \\
\hline 2025 & mujer & 5.45 & 4.63 & 5.34 \\
\hline 2220 & rostro pensativo & 5.14 & 5.03 & 4.97 \\
\hline 2270 & niño & 5.34 & 4.14 & 5.64 \\
\hline 2394 & médica & 5.29 & 4.67 & 5.11 \\
\hline 2410 & niño sumando & 5 & 4.54 & 4.95 \\
\hline 2635 & vaquero & 5.4 & 4.68 & 4.93 \\
\hline 3005.2 & oro enterrado & 5.46 & 5.5 & 5.38 \\
\hline 4233 & prostituta & 5 & 5.05 & 5.08 \\
\hline 4320 & mujer sensual & 5.49 & 5.34 & 4.98 \\
\hline 4503 & hombre erótico & 5.48 & 5.03 & 5.19 \\
\hline 5950 & rayos & 5.15 & 6.31 & 3.42 \\
\hline 7095 & faro & 5.45 & 4.52 & 5.41 \\
\hline 7130 & camión & 5.24 & 4.17 & 5.2 \\
\hline 7504 & escalera & 5.07 & 4.56 & 5.44 \\
\hline 7510 & rascacielos & 5.17 & 4.99 & 4.4 \\
\hline 8116 & fútbol americano & 5.28 & 5.16 & 5.46 \\
\hline 8160 & montaña & 5.41 & 6.68 & 4.45 \\
\hline 8192 & esquiador volcán & 5.07 & 7.08 & 4.99 \\
\hline media & & 5.25 & 5.22 & 4.93 \\
\hline
\end{tabular}

\begin{tabular}{llrrr}
\hline FOTO N & \multicolumn{1}{c}{ DESCRIPCIÓN } & $\mathrm{V}^{*}$ & $\mathrm{~A}^{*}$ & $\mathrm{D}^{*}$ \\
\hline DESAGRADABLES & & & \\
2205 & hospital & 1.84 & 5.79 & 2.62 \\
2276 & chica llorando & 2.29 & 5.8 & 3.56 \\
2455 & niñas tristes & 2.59 & 5.59 & 3.82 \\
2750 & alcohólico & 2.25 & 5.92 & 3.8 \\
2751 & conductor borracho & 2.57 & 5.88 & 4.19 \\
2795 & chico & 2.39 & 5.96 & 3.63 \\
3180 & mujer apaleada & 2.14 & 3.71 & 3.19 \\
3220 & hospital & 2.37 & 5.85 & 3.45 \\
6241 & pistola & 2.71 & 5.71 & 3.59 \\
6311 & mujer triste & 2.72 & 5.92 & 4.17 \\
6830 & armas & 2.54 & 5.93 & 3.1 \\
9041 & niño asustado & 2.13 & 5.86 & 3.65 \\
9220 & cementerio & 2.09 & 4.71 & 2.81 \\
9300 & inodoro sucio & 2.54 & 5.24 & 4.28 \\
9342 & contaminación & 2.43 & 6.02 & 3.51 \\
9415 & discapacitado & 1.89 & 5.77 & 2.91 \\
9470 & ruinas & 2.69 & 5.56 & 2.98 \\
9471 & edificio quemado & 2.51 & 5.83 & 3.28 \\
9571 & gato putrefacto & 2.09 & 5.9 & 3.68 \\
9911 & accidente coche & 2.25 & 5.87 & 2.86 \\
media & & 2.35 & 5.64 & 3.45 \\
\hline & & & &
\end{tabular}

*Puntuaciones de: $\mathrm{V}=$ valencia, $\mathrm{A}=$ arousal, $\mathrm{D}=$ dominancia 
